\title{
TRANSIENT GAS MOTION IN NUCLEAR-REACTOR-PUMPED LASERS: \\ COMPUTATIONAL AND EXPERIMENTAL RESULTS
}

J. R. Torczynski

Senior Member of the Technical Staff

Fluid, Thermal, and Structural Sciences Department

Sandia National Laboratories

Albuquerque, New Mexico 87185-5800

SAND- $-91-1441 \mathrm{C}$

and

DE92 002357

D. R. Neal

Senior Member of the Technical Staff

Laser and Chemical Physics Research Department

Sandia National Laboratories

Albuquerque, New Mexico 87185-5800

\section{ABSTRACT}

In some types of nuclear-reactor-pumped lasers, the fission fragments that are used to excite gaseous lasing species heat the gas in a spatially nonuniform manner. This heating nonuniformity induces transient gas motion, which results in density and refractive-index gradients that affect the laser's optical behavior. A model of the transient gas motion is developed using the acoustic filtering methodology, which neglects the spatial variation of the pressure. This model incorporates the effect of spatially varying gas density on fissionfragment heating. Gas motion out of the laser cell into small, rapidly cooled regions is treated as a volumetric mass loss distributed over the laser cell. Although these regions have a relatively small fraction of the total volume, a large amount of gas can flow into them during the heating because of the rapid cooling therein. This gas removal from the cell during pumping, neglected in previous analyses, is important because fission-fragment heating is strongly dependent on local gas density. To quantify the laser's optical behavior, experiments are performed in which a probe laser beam is passed through the laser cell. This probe beam acquires a wavefront distortion from the refractive-index gradients and is imaged onto a wavefront slope sensor, which yields temporally and spatially resolved measurements of the angular deflection (wavefront slope) of the probe laser beam. Experimental and model results for this quantity exhibit reasonably good agreement over a wide range of pressures and heating amplitudes.

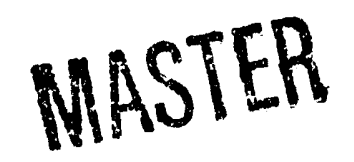


NOMENCLATURE

\begin{tabular}{|c|c|}
\hline$A$ & neutron flux amplitude \\
\hline$c_{p}$ & specific heat \\
\hline$L_{x}$ & cell half-width \\
\hline$L_{3}$ & model cell length (pumped plus cooled) \\
\hline$f$ & heating spatial variation \\
\hline $\boldsymbol{F}$ & lenslet focal length \\
\hline$h$ & heating temporal variation \\
\hline $\boldsymbol{k}$ & thermal conductivity \\
\hline$p$ & pressure \\
\hline$Q$ & volumetric heating \\
\hline$R$ & gas constant \\
\hline$t$ & time \\
\hline$t_{p}, t_{s}$ & neutron flux time constants \\
\hline$t_{j}$ & time $j$ of data frame $j$ \\
\hline$T$ & temperature \\
\hline$u$ & velocity $x$ component \\
\hline$x, y, z$ & spatial coordinates \\
\hline$x_{i j}$ & focal spot position $i$ at time $j$ \\
\hline$\gamma$ & specific heat ratio \\
\hline$\Lambda$ & expansion rate \\
\hline$\rho$ & density \\
\hline$\theta_{i j}$ & exit angle $i$ at time $j$ \\
\hline$\chi$ & rapidly cooled volume fraction \\
\hline$\overline{(})$ & $x$-averaged () \\
\hline()$_{m}$ & maximum () \\
\hline()$_{r}$ & () in rapidly cooled region \\
\hline()$_{0}$ & initial or scale () \\
\hline
\end{tabular}

\section{DISCLAIMER}

This report was prepared as an account of work sponsored by an agency of the United States Government. Neither the United States Government nor any agency thereof, nor any of their employees, makes any warranty, express or implied, or assumes any legal liability or responsihility for the accuracy, completeness, or usefulness of any information, apparatus, product, or process disclosed, or represents that its use would not infringe privately owned rights. Referprocess disclosed, or represe commercial product, process, or service by trade name, trademark, ence herein to any specifice does not necessarily constitute or imply its endorsement, recommanufacturer, or otherwise doe United States Government or any agency thereof. The views and opinions of authors expressed herein do not necessarily state or reflect those of the Innited States Government or any agency thereof. 


\section{INTRODUCTION}

If fissionable material is irradiated by neutrons from a nuclear reactor, the fission fragments thus produced can be used directly to excite (pump) atomic and molecular gaseous lasing species. Such a direct excitation scheme (fission-fragment energy to excited lasing species) obviates the need for intermediate energy-conversion steps (fission-fragment energy to heat, heat to electricity, electricity to pumping scheme, pumping scheme to excited lasing species) and thus offers the possibility of greater lasing efficiency (Miley, 1970). Nuclearreactor-pumped lasers were first observed experimentally by McArthur and Tollefsrud (1975) and have been studied by many investigators since then (see McArthur (1991) for an excellent review of this field). However, the presence of refractive-index gradients caused by heating-induced gas motion in the laser cell has hampered the development of these lasers (Neal et al., 1989a,1989b).

Refractive-index gradients within the laser cell arise in the following manner. In typical nuclear-reactor-pumped lasers, the fission fragments enter the gas from thin coatings of fissionable materiai on the side walls of the laser cell, which are parallel to the optical axis. Although some of the fission-fragment energy that is deposited in the gas is converted to laser light, the bulk of this energy appears as heat. This heating is spatially nonuniform because the fission fragments enter the gas from the side walls. As a result, the heating is largest near the side walls and smallest in the center of the laser cell. Another source of spatial nonuniformity is the removal of heat from the gas near the side walls by thermal conduction to the walls, which remain essentially unheated during pumping. In addition to being nonuniform, the heating of the gas is large in the sense that the induced pressure rise is comparable to the gas pressure prior to heating. Since the dimensions of the laser cell are typically much smaller than the distance that an acoustic wave can travel while the energy is being deposited, the heating spatial nonuniformity can induce significant gas motion and cross-axis density gradients within the laser cell (see Fig. 1) during the pumping (Torczynski, 1989). Unlike many commion lasers, nuclear-reactor-pumped lasers typically 
operate with the gas near room temperature and near or above atmospheric pressure. As a result, the cross-axis refractive-index gradients are strong enough to affect the laser's optical behavior. In fact, under certain circumsinirces, the lensing induced in this manner can alter the stability properties of the laser resonator, as observed by Neal et al. (1989a,1989b,1990).

To quantify the gas motion in nuclear-reactor-pumped lasers and its effect on optical behavior, two approaches are followed herein. First, a computational model is developed that describes the heating-induced gas motion. This model is formulated using the acoustic filtering methodology of Rehm and Baum (1978), which neglects the spatial variation of the pressure within the laser cell. The coupling between the gas motion and the heating, which results both from the gas-density dependence of the fission-fragment heating (Torczynski, 1989) and the heat transfer out of the gas to the side walls, is included in the model. Gas motion out of the laser cell and into small, rapidly cooled regions is also incorporated into the model. This effect, neglected in previous analyses (Torczynski and Gross, 1988; Torczynski et al., 1989), is found to be important for many experiments. Despite the fact that these regions have a relatively small fraction of the total volume, a large amount of gas can flow out of the cell and into these regions during the heating, which in turn reduces the fission-fragment heating. Second, experiments are performed in which a probe laser beam passing through the laser cell acquires a wavefront distortion from the refractive-index gradients. This probe beam is subsequently imaged onto a wavefront slope sensor, which yields temporally and spatially resolved measurements of the angular deflection (wavefront slope) of the probe laser beam. The experimental measurements thus probe the temporal and spatial variation of the gas density field (integrated along the optical axis) during pumping.

The computational model is used to analyze the six sets of conditions at which experimental results are reported. Pressure histories and angular deflections are compared, and model limitations are discussed based on this comparison. Model resulis for the temporal dependence of the effective focal length and the effective aperture of the laser cell (see Fig. 1) are also presented. 


\section{COMPUTATIONAL MODEL}

In the development of a computational model to simulate the response of gas in a laser cell to fission-fragment heating, it is necessary to understand the two principal characteristics that are desirable for the model. First, the model should be capable of reproducing relevant observed quantities. Examples include the pressure history during and after the pumping and the spatial and temporal variation of the angular deflection of the probe beam during the pumping (but not necessarily after), which requires knowledge of the time variation of cross-axis density and refractive-index gradients. Second, the model should be of relatively low computational intensity so that it can be used to perform parameter studies over a wide range of conditions. This latter characteristic suggests treating important physical phenomena accurately while approximating phenomena of minor importance, where phenomena are deemed important herein if they significantly affect experimentally observed quantities.

In the spirit of this approach, the model geometry (see Fig. 2) is simplified considerably from the three-dimensional experimental geometry and consists of two distinct regions, between which gas is exchanged. The pumped region is geometrically identical to the pumped region of the experimental laser cell and has length $\left(1-\chi_{0}\right) L_{z}$, half-width $L_{x}$, and volume fraction $1-\chi_{0}$, with symmetry assumed about the centerplane $(x=0)$. In this region, quantities such as the density $\rho$, the temperature $T$, and the cross-axis velocity component $u$ are permitted to depend only on $x$ and $t$ (not on $y$ or $z$ ). The rapidly cooled region, with volume fraction $\chi_{0}$, represents the lumped effect of all the extraneous regions like fill lines, gaps between optical components, etc., which are typically located at the end of the experimental laser cell. In this region, quantities such as the density $\rho_{r}$ and the temperature $T_{r}$ are permitted to depend only on $t$. Although at first glance these assumptions seem severe, the pumped region of the experimental laser cell is rather one-dimensional (no $y$ or $z$ variation) since aspect ratios are usually large $\left(\left(1-\chi_{0}\right) L_{z} / L_{x} \sim O\left(10^{2}\right)\right)$ and the neutron flux inducing the heating typically does not vary significantly with $y$ or $z$. The rapidly cooled region acts principally as a mass sink during pumping, taking up gas that 
has expanded out of the pumped region, and as a mass source afterward, compressing the heated gas that has remained in the pumped region, and as such does not significantly alter the one-dimensionality. Furthermore, the probe laser beam does not resolve variation in $z$ but instead averages along the $z$ direction.

To model the gas, the following assumptions are made. The gas is a mixture of perfect gases that obeys the perfect gas equation of state

$$
p=R \rho T
$$

and has constant specific heats and specific heat ratio $\gamma$. The thermal conductivity $k$ is described by the empirical relation (Torczynski, 1990a)

$$
k=k_{0}\left(T / T_{0}\right)^{3 / 4}
$$

where $k_{0}$ is determined for the gas mixture at $T_{0}$ following Reid et al. (1986).

The fission-fragment heating $Q$ is of the coupled form

$$
Q=Q_{0} f[x ; \rho] h(t)
$$

where $Q_{0}$ is the heating amplitude, $h$ is the normalized temporal dependence of the neutron flux, and $f$ describes the spatial variation of the heating. More specifically, $f$ has a minimum at the centerplane $(x=0)$, is largest at the side walls $\left(x= \pm L_{x}\right)$, and depends strongly on the density field in a nonlocal fashion (molecules at a point absorb energy from the flux reaching that point), as discussed by Torczynski (1989).

Heat is removed from the gas in the following manner. In the pumped region, thermal conduction removes heat from the gas to the side walls, which are held at the initial temperature $T_{0}$. In the rapidly cooled region, heat transfer from the gas operates on a time scale much shorter than the pumping duration, so the gas in this region is taken to remain at temperature $T_{r}=T_{0}$. Following pumping, this gas is allowed to expand isothermally back into the pumped region. Thus, during pumping, the rapidly cooled region acts like an isothermal mass sink, here applied to all points in the pumped region as a uniform expansion 
rate, whereas following pumping, the gas in the rapidly cooled region compresses the heated gas at a uniform rate as the heated gas cools.

The transient gas flow in the pumped region has speeds that are low compared to the gas sound speed, which permits the equations of motion to be acoustically filtered following the approach of Rehm and Baum (1978). In this approach, the thermodynamic pressure $p$ depends only on $t$, not on position (Torczynski, 1989), yielding equations of the form (Torczynski, 1990b)

$$
\begin{gathered}
\frac{\partial \rho}{\partial t}+\frac{\partial}{\partial x} \rho u+\rho \Lambda=0 \\
\frac{d p}{d t}+\gamma p \Lambda=(\gamma-1)\left[\bar{Q}+\overline{\left.\frac{\partial}{\partial x}\left(k \frac{\partial T}{\partial x}\right)\right]}\right. \\
\gamma p \frac{\partial u}{\partial x}=(\gamma-1)\left[Q-\bar{Q}+\frac{\partial}{\partial x}\left(k \frac{\partial T}{\partial x}\right)-\overline{\frac{\partial}{\partial x}\left(k \frac{\partial T}{\partial x}\right)}\right]
\end{gathered}
$$

where terms with an overbar are averaged over $x$. The quantity $\Lambda$ is the average expansion rate in the pumped region that is caused by flow into the rapidly cooled region and is taken to depend only on $t$. As discussed above, the quantities $\rho, T, u$, and $Q$ depend only on $x$ and $t$.

To close this system of equations, models for the expansion rate $\Lambda$ are needed for the two different situations of flow out of or into the pumped region. In these two situations, the rapidly cooled region behaves slightly differently, as discussed above, although it is always the case that

$$
p=R \rho_{r} T_{0}
$$

In the first situation, the model for $\Lambda$ must reflect the fact that the mass flow out of the pumped region must equal the mass flow into the rapidly cooled region so that the total mass in the system remains fixed. Equal mass flows require that

$$
\chi_{0} \frac{d \rho_{r}}{d t}=\bar{\rho}\left(1-\chi_{0}\right) \Lambda
$$

where $\bar{\rho}$ is the average density in the pumped region, so the right-hand side of this expression represents outflow from the pumped region at the conditions in the pumped region. Fixing 
the total mass in the system requires that

$$
\left(1-\chi_{0}\right) \bar{\rho}+\chi_{0} \rho_{r}=\rho_{0},
$$

where $\rho_{0}$ is the initial density. Equations (7)-(9) can be combined to yield

$$
\Lambda=\left(\frac{\chi_{0}}{p_{0}-\chi_{0} p}\right) \frac{d p}{d t} .
$$

Note that this relation implies that the pressure always remains less than $p_{0} / \chi_{0}$.

In the second situation, the model for $\Lambda$ must reflect the fact that, after the pressure has reached its maximum value, the gas in the rapidly cooled region, originally of extent $\chi_{0}$, expands isothermally back into the pumped region, compressing the gas in the pumped region. As a result, the volume fraction $\chi$ of gas with $T=T_{0}$ increases with time, so the boundary in Fig. 2 moves leftward as the pressure falls. This admittedly crude characterization of the refilling process during cooling is not able to produce accurate cross-axis density profiles; nevertheless, it will be shown below to reproduce pressure histories with reasonable accuracy. Since mass does not flow across the expanding boundary, mass conservation and isothermality imply that

$$
\frac{p}{p_{m}}=\frac{\rho_{r}}{\rho_{r, m}}=\frac{\chi_{0}}{\chi}
$$

where $p_{m}$ is the pressure maximum and $\rho_{r, m}$ is the maximum density in the rapidly cooled region. The definition of $\Lambda$ as the expansion rate (or, equivalently, continuity of velocity at the moving boundary) requires that

$$
\frac{d}{d t}(1-\chi)=(1-\chi) \Lambda
$$

These equations can be combined to yield

$$
\Lambda=\left(\frac{\chi_{0} p_{m} / p}{p-\chi_{0} p_{m}}\right) \frac{d p}{d t} .
$$

Note that Equations (10) and (13) both imply vanishing $\Lambda$ at the pressure maximum. During the period immediately following the attainment of the pressure maximum, the rate of pressure decrease car be roughly estimated. Since the thickness of a transient thermal boundary 
layer produced by a heating pulse of duration $t_{\mathrm{s}}$ scales as $\sqrt{k_{0} t_{0} / \rho_{0} c_{p}}$, where $c_{p}=\gamma R /(\gamma-1)$ is the specific heat, Equations (1), (2), (5), (9), (11), and (13) imply the relation

$$
\frac{d p}{d t} \sim-\left(1+\frac{\gamma \chi_{0}}{1-\chi_{0}}\right)^{-1}\left(\frac{\gamma p_{0}}{t_{m}}\right)\left[\frac{\left(1-\chi_{0}\right)\left(p_{m} / p_{0}\right)}{1-\chi_{0}\left(p_{m} / p_{0}\right)}\right]^{7 / 4},
$$

where it is assumed that $p \sim p_{m}$ and that $\bar{Q}$ is small following pumping. The term on the right-hand side of Equation (14) is seen to increase dramatically as the ratio $p_{m} / p_{0}$ is increased. Therefore, low-pressure experiments, which by definition have large values of $p_{m} / p_{0}$, are expected to exhibii unusually rapid pressure decays when compared with highpressure experiments.

Equations (10), (13), and (4)-(6), together with the fission-fragment model for $f[x ; \rho]$ discussed by Torczynski (1989), form a closed system of equations describing the gas motion which are solved by conventional finite-difference techniques. Once the density field is determined, tive relation $n=1+\left(n_{0}-1\right) \rho / \rho_{0}$ yields the refractive-index field $n$ in the gas. Ray-tracing analyses, like those of Torczynski and Gross (1988), are performed from $z=0$ to $z=\left(1-\chi_{0}\right) L_{z}$ (the extent of the pumped region) using the computed refractive-index field for the pumped region. The analysis of the experiments discussed in the next section is based on this approach.

\section{EXPERIMENTAL METHOD}

To understand the effect of gas motion on the optical behavior of a nuclear-reactorpumped laser, it is necessary to characterize the refractive-index field that exists in the gas as it is pumped. An experimental characterization requires simultaneous measurements of the refractive index at enough cross-axis locations in the laser cell to reconstruct its cross-axis variation. Since the field is not static, such measurements are required at many times throughout the pumping. Although an array of probes placed within the laser cell at locations spanning its width could in principal measure a quantity such as temperature, from which density and refractive index values could be inferred, the invasive nature of such measurements, the need for highly time-resolved data, and the deleterious effect of the harsh environment within a nuclear reactor on electronics renders this option impractical. Optical 
techniques, however, are well suited to this task. First, the refractive-index field, which is the quantity of fundamental interest, can be probed directly by passing light through the gas in the laser cell. Second, such a measurement provides field information, rather than information at discrete locations although an integration of the field along ray paths does occur. Third, the data acquisition rate is limited essentially only by the rate at which data can be recorded. Fourth, sampling the refractive-index field in this manner is a noninvasive measurement and is largely unaffected by the harsh nuclear environment.

Neal et al. (1991) recently reported the development of a Hartmann-Shack wavefront slope (ray angular deflection) sensor which can measure the spatial and temporal variation of the slope of an optical wavefront entering the sensor. The wavefront slope sensor consists of a CCD carnera with 2048 elements and an array of lenslets covering the CCD camera aperture (see Fig. 3). The lenslets are separated by a center-to-center distance of $2.54 \mathrm{~mm}$ and are focused on the CCD array. The CCD camera data (focal spot positions) are recorded with a synchronous 12-bit transient digitizer. In this configuration, the wavefront slope sensor has a maximum frame rate of $20 \mathrm{kHz}$, a dynamic range of $\pm 10 \mathrm{mrad}$, and a resolution of 0.0003 mrad. This resolution is far smaller than the angular extent of a single element of the CCD array and is obtained by use of a centroid-determination algorithm to find the position of the focal spot's center (Neal et al., 1991). Provisions for image magnification are also included so that the sensor is not constrained to examine fields with sizes similar to its aperture.

Wavefront slopes, which are normal to ray angular deflections, are determined in the following manner (see Fig. 3), where here it is assumed for simplicity that the refractiveindex gradients modifying the wavefront vary in $x$ but not in $y$. The angle $\theta_{i j}$ of the ray entering lenslet $i$ at time $t_{j}$ determines the position of the focal spot according to the relation $\theta_{i j}=\left(x_{i j}-x_{i 0}\right) / F$, where $F$ is the lenslet focal length and $x_{i 0}$ is the reference focal spot position. The wavefront slope sensor can be used in two different modes. In the absolute mode, an absolute reference is used to assign a value to the $x_{i 0}$, whereas in the relative 
mode, the $x_{i 0}$ are typically chosen to be the focal spot positions prior to the introduction of refractive-index gradients. Thus, the wavefront slope sensor data allow determination of the ray angular deflections (wavefront slopes) as functions of time at various fixed positions; furthermore, spatial integration of these slopes permits determination of the wavefront shape entering the sensor at each time $t_{j}$ if desired (Neal et al., 1991).

The wavefront slope sensor is used in the following manner to characterize the refractiveindex field in the laser cell. A probe laser beam is expanded, collimated, and passed through the laser cell parallel to the optical axis. As the beam traverses the laser cell, the refractiveindex gradients induce beam steering of the rays comprising the beam (see Fig. 1), so the angle $\theta$ of a ray exiting the laser cell is a function of the position $x$ at which it exits the laser cell. To determine the variation of the exit angle with exit position at the exit plane of the laser cell, the exit plane is imaged onto the wavefront slope sensor. Since imperfections in the experimental optical system introduce small steady-state angular deflections into the probe laser beam, either the sensor is used in the relative mode or a calibration is performed immediately before each use. For the experiments discussed in the following section, typically about 125 frames of data at $0.1 \mathrm{mrad}$ resolution are recorded from the wavefront slope sensor per experiment over a time interval of $80-90 \mathrm{~ms}$.

\section{MODEL AND EXPERIMENTAL RESULTS}

Six sets of conditions are selected for comparison of model and experimental results (see Table 1). The laser cell used in the experiments has dimensions $1.5 \mathrm{~cm} \times 7 \mathrm{~cm} \times 60 \mathrm{~cm}$, and the rapidly cooled volume fraction of this cell is $15 \%$. These quantities correspond to model parameter values of $\chi_{0}=0.15, L_{x}=0.15 \mathrm{~cm}$, and $L_{z}=70.6 \mathrm{~cm}\left(\left(1-\chi_{0}\right) L_{z}=60 \mathrm{~cm}\right)$. In all cases, the initial gas temperature is $T_{0}=300 \mathrm{~K}$, the specific heat ratio is $\gamma=5 / 3$, and the initial gas pressure $p_{0}$ is the sum of the partial pressures shown in Table 1. In the experiments, the heating is produced by $0.91-\mu \mathrm{m} \mathrm{UO_{2 }}$ foils on the $7 \mathrm{~cm} \times 60 \mathrm{~cm}$ side walls, which are subjected to two different neutron pulses, a "weak" pulse and a "strong" pulse. The neutron-flux histories for these pulses are shown in Fig. 4, from which it is seen that 
Table 1. Experimental Conditions

\begin{tabular}{|c||c|c|c|}
\hline Expt & Pulse & $p_{\text {Ar }}(\mathrm{kPa})$ & $p_{\text {Xe }}(\mathrm{kPa})$ \\
\hline A4435 & weak & 51.7 & 0.259 \\
A4438 & weak & 103.4 & 0.517 \\
\hline A4477 & strong & 51.7 & 0.259 \\
A4476 & strong & 103.4 & 0.517 \\
A4474 & strong & 172 & 0.862 \\
A4473 & strong & 414 & 2.068 \\
\hline
\end{tabular}

the strong pulse has five times the amplitude of the weak pulse but lasts only half as long. In the model, the temporal variation of the neutron flux is approximated by the Gaussian form $h(t)=\exp \left\{-\left[\left(t-t_{p}\right) / t_{s}\right]^{2}\right\}$. Table 2 shows the best-fit values for the parameters of the Gaussian $A h(t)$, and the model neutron-flux histories are shown in Fig. 4 for comparison. Also shown in Table 2 is the heating amplitude $Q_{0}$ (the neutron-flux amplitude $A$ multiplied by the conversion factor $7.11 \times 10^{-5}$ ).

Table 2. Pumping Parameters

\begin{tabular}{|c||c|c|c|c|}
\hline Pulse & $t_{p}(\mathrm{~ms})$ & $t_{\circ}(\mathrm{ms})$ & $A$ (arb. units) & $Q_{0}\left(\mathrm{~kW} / \mathrm{cm}^{3}\right)$ \\
\hline weak & 75.06 & 9.13 & 365 & 0.026 \\
strong & 52.30 & 4.50 & 1833 & 0.130 \\
\hline
\end{tabular}

In Fig. 5, the computational and experimental pressure histories are compared for experiments A4438, A4477, and A4476. The experimenial pressure measurements are made using a Kulite pressure transducer with a $1 \mathrm{kHz}$ response that is mounted near the end of the laser cell. Also included for comparison in Fig. 5 are computational results for cases identical to the above except that no rapidly cooled region is included (equivalent to taking $\chi_{0}=0$ ). In the computations with $\chi_{0}=0.15$, the rate of pressure increase during pumping, the peak pressure that is obtained, and the rate of pressure decrease following the pumping are all predicted with reasonable accuracy. The computed pressure histories with $\chi_{0}=0$, however, differ by as much as a factor of 2 in pressure rise from the experimental results, so it is seen that a modest-size rapidly cooled region (15\%) exerts a disproportionately large effect on 
the pressure rise. Note that the low-pressure experiments A4476 and A4477 have similar large values of $d p / d t$ following the pressure maximum, whereas the medium-pressure experiment A4438 has a significantly lower value, as predicted by Equation (14). Although not shown, the other tinree cases, which span significant ranges of initial pressures, heciling rates, and heating amplitudes, exhibit comparably good agreement when the value $\chi_{0}=0.15$ is used. Since the removal of gas from the pumped region significantly affects the pressure rise through the density-dependence of fission-fragment heating, the agreement of peak pressures and rate of pressure rise is good evidence that the flow of gas out of the pumped region is accurately described. Similarly, the agreement following pumping indicates that the relatively crude approximations used to describe this period of time are adequate, at least for describing the pressure history. However, these pressure bistories in and of themselves give no indication as to how accurately the cross-axis gas motion is described.

The dependence of the exit angle $\theta$ of the probe laser beam on the exit position $x$ (see Fig. 1) is shown at a series of times for experiment A4435 in Fig. 6. The comparison times span the duration of the pumping, which corresponds essentially to the period during which the pressure in the laser cell is rising. From this comparison, it is seen that the model predicts the spatial and temporal dependence of the angular deflection of the probe laser beam with reasonable accuracy throughout the duration of the pumping pulse for this experiment. This implies that the refractive-index field and hence the density field are predicted with similar accuracy, indicating that the strong coupling that exists between the fission-fragment heating and the cross-axis gas motion (Torczynski, 1989) is correctly described by the model. Additional angular-deflection comparisons are shown for the other experiments at selected times in Figs. 7-11. Note that in all cases the overall amplitude of the angular deflection is predicted reasonably well despite varying substantially from one case to another. For each case, however, the model overpredicts the experimental observations slightly. The level of agreement between experiment and model is seen to degrade somewhat as the ratio $p_{0} / p_{m}$ decreases: predictions for the low-pressure, strong-pulse experiment A4477 are the least 
accurate. Given the above indications of the model's accuracy, the model can be used to determine two quantities of importance that characterize the laser's optical behavior: the effective focal length and the effective aperture of the pumped region (see Fig. 1). Plots of these quantities are shown as functions of time for four experiments in Fig. 12. In some cases, focal lengths and apertures as small as $20 \mathrm{~cm}$ and $0.5 \mathrm{~cm}$, respectively, are observed.

\section{CONCLUSIONS}

To study the transient gas motion that occurs within a nuclear-reactor-pumped laser, a computational model has been developed, and experimental data have been obtained, enabling assessment of the model's accuracy. While simplifying the geometry considerably, the model incorporates accurate descriptions of the strong coupling between the cross-axis gas motion and the fission-fragment heating and of the gas flow out of the laser cell into small, rapidly cooled regions. To determine the temporal and spatial variation of the refractiveindex field in the laser cell, a recently developed sensor that measures the slope of an optical wavefront is employed in the experiments. A comparison of model and experimental results indicates reasonable agreement over significant ranges of the gas pressure and the pumping amplitude. Pressure histories are found to agree closely both during the pumping and afterward, so long as the effect of the small ( $15 \%$ volume fraction) rapidly cooled region is included; furthermore, neglect of this effect is shown to produce pressure rises differing from the experimental results by roughly a factor of 2 . The spatial and temporal variation of the angular deflection of the probe laser beam is also found to be in reasonable agreement for all of the cases examined. However, the model exhibits a slight overprediction of the angular deflection, which is most significant for experiments with low initial pressures compared to the maximum pressure. Nevertheless, the model is sufficiently accurate to predict the effective focal length and effective aperture of the laser cell throughout pumping, both of which strongly influence the laser's optical behavior and are used to categorize experimentally observed abrupt variations in the time-dependent laser intensity as resulting either from the motion-induced refractive-index gradients (laser resonator stability transitions) or from the 
kinetics of the lasing species (temperature effects). Although a specific application of the wavefront slope sensor is discussed in this paper, this technique is of broad applicability ar:d can be used to characterize a wide variety of steady and transient flows with refractive-index variations. Furthermore, this technique has an advantage over many optical techniques: accurate quantitative results are obtained for density gradients in the medium through which the probe beam has passed.

\section{ACKNOWLEDGMENTS}

The authors gratefully acknowledge many helpful interactions with R. B. Michie, W. J. Alford, W. C. Sweatt, D. E. Bodette, D. A. McArthur, and A. J. Suo-Anttila, all of Sandia National Laboratories. The nuclear-reactor experiments involve the efforts of numerous individuals at Sandia, whose enthusiastic cooperation and hard work are greatly appreciated by the authors. This work was performed at Sandia National Laboratories, supported by the U. S. Department of Energy under contract number DE-AC04-76DP00789.

\section{REFERENCES}

McArthur, D. A., and Tollefsrud, P. B., 1975, "Observation of Laser Action in CO Gas Excited Only by Fission Fragments," Appl. Phys. Lett. Vol. 26, pp. 187-190.

McArthur, D. A., 1991, "Nuclear Pumped Lasers," Encyclopedia of Lasers and Optical Technology, R. A. Meyers, ed., Academic Press, New York, pp. 385-393.

Miley, G. H., 1970, Direct Conversion of Nuclear Radiation Energy, American Nuclear Society, LaGrange IL, Chaps. 3-4.

Neal, D. R., Sweatt, W. C., and Torczynski, J. R., 1989a, "Resonator Design with an Intracavity Time-Varying Index Gradient," SPIE Paper 965-40, Current Developments in Optical Engineering III, R. E. Fischer and W. J. Smith, eds., SPIE Proceedings, Vol. 965, pp. 130-141.

Neal, D. R., Torczynski, J. R., and Sweatt, W. C., 1989b, "Resonator Stability Effects in "Quadratic-Duct" Nuclear-Reactor-Pumped Lasers," Proceedings of the International Conference on Lasers '88, R. C. Sze and F. J. Duarte, eds., STS Press, McLean VA, pp. 245-252. 
Neal, D. R., Torczynski, J. R., and Sweatt, W. C., 1990, "Time-Resolved Wavefront Measurements and Analyses for a Pulsed, Nuclear-Reactor-Pumped Gain Region," Optical Engineering, Vol. 29, No. 11, pp. 1404-1412.

Neal, D. R., Torczynski, J. R., Alford, W. J., Michie, R. B., and Bodette, D. E., 1991, "Time-Dependent Wavefront Error Measurements for a Long-Pulse Wall-Pumped Laser," presented at the 1991 Conference on Lasers and Electro-Optics.

Rehm, R. G., and Baum, H. R., 1978, "The Equations of Motion for Thermally Driven, Buoyant Flows," Journal of Research of the National Bureau of Standards, Vol. 83, pp. 297308.

Reid, R. C., Prausnitz, J. M., and Poling, B. E., 1986, The Properties of Gases and Liquids, McGraw-Hill, New York, pp. 530-531.

Torczynski, J. R., 1989, "On the motion of a gas experiencing range-dependent volumetric heating," Journal of Fluid Mechanics, Vol. 201, pp. 167-188.

Torczynski, J. R., 1990a, "Heat-Transfer Regimes in Nuclear-Reactor-Pumped Gas Lasers," General Papers: Phase Change and Convective Heat Transfer, HTD-Vol. 129, K. Vafai, M. A. Ebadian, T. Diller, M. K. Jensen, T. W. Simon, A. G. Lavine, and W. Aung, eds., American Society of Mechanical Engineers, New York, pp. 111-118.

Torczynski, J. R., 1990b, "Partial Acoustic Filtering Applied to the Equations of Compressible Flow, ${ }^{n}$ International Symposium on Nonsteady Fluid Dynamics, FED-Vol. 92, J. A. Miller and D. P. Telionis, eds., American Society of Mechanical Engineers, New York, pp. 201-206.

Torczynski, J. R., and Gross, R. J., 1988, "The Gasdynamics of Fission-Fragment Heating," Proceedings of the 1st National Fluid Dynamics Congress, C. Dalton, ed., AlAA, Washington DC, pp. 1040-1047.

Torczynski, J. R., Gross, R. J., Hays, G. N., Harms, G. A., Neal, D. R., McArthur, D. A., and Alford, W. J., 1989, "Fission-Fragment Energy Deposition in Argon," Nuclear Science and Engineering, Vol. 101, No. 3, pp. 280-284. 


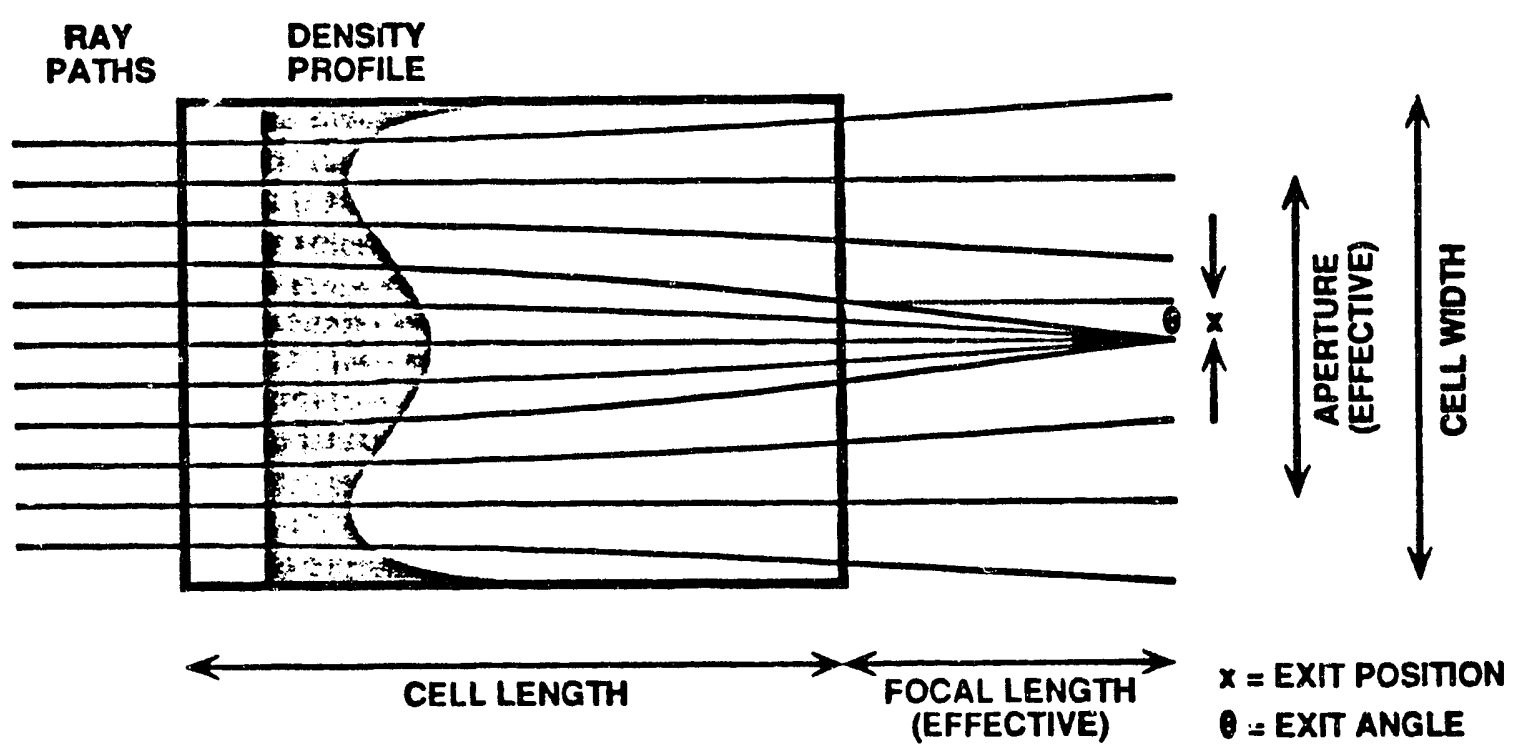

Fig. 1 Schematic diagram of a nuclear-reactor-pumped laser cell (aspect ratio greatly reduced) showing the density profile that produces lens-like behavior. The angular deflection $\theta$ is measured as a function of the distance $x$ from the centerplane of symmetry.
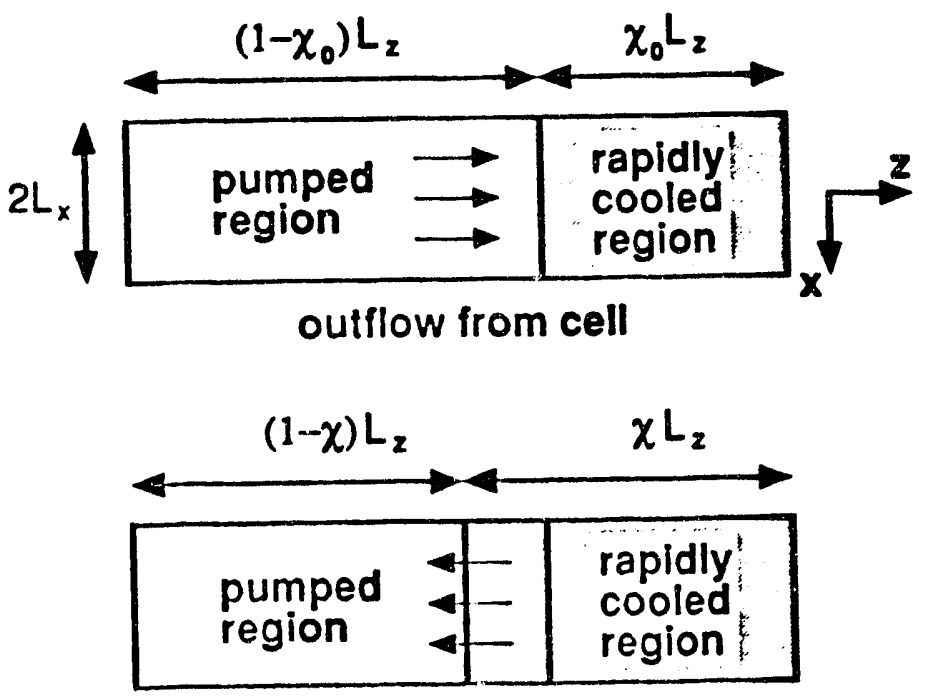

inflow to cell

Fig. 2 Model geometry: rapidly cooled regions are lumped together and treated as an isothermal volumetric mass sink. 


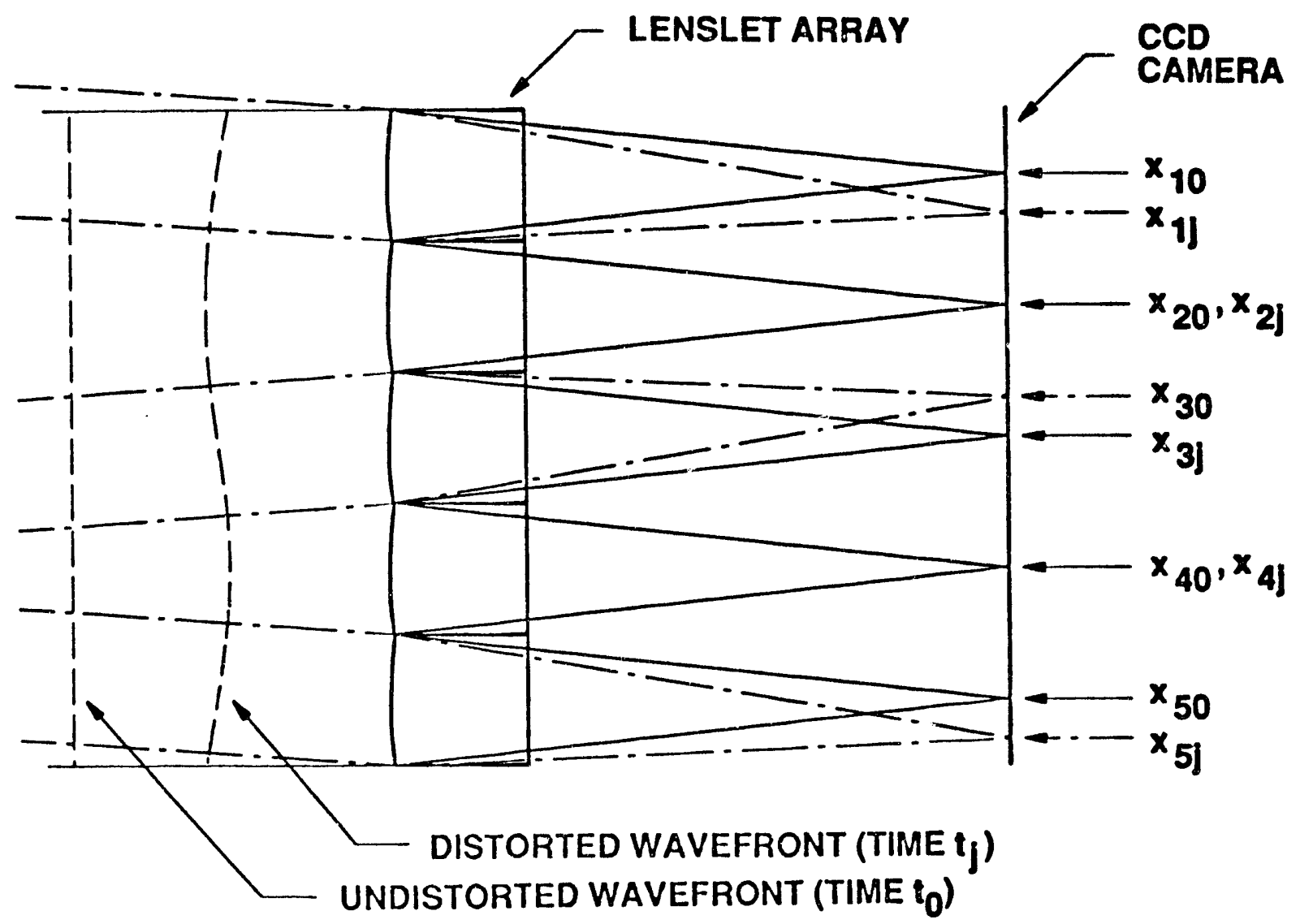

Fig. 3 Wavefront slope sensor operation: wavefront slope $\theta_{i j}$ (ray angle) is recorded as focal spot displacement $x_{i j}-x_{i 0}$. 

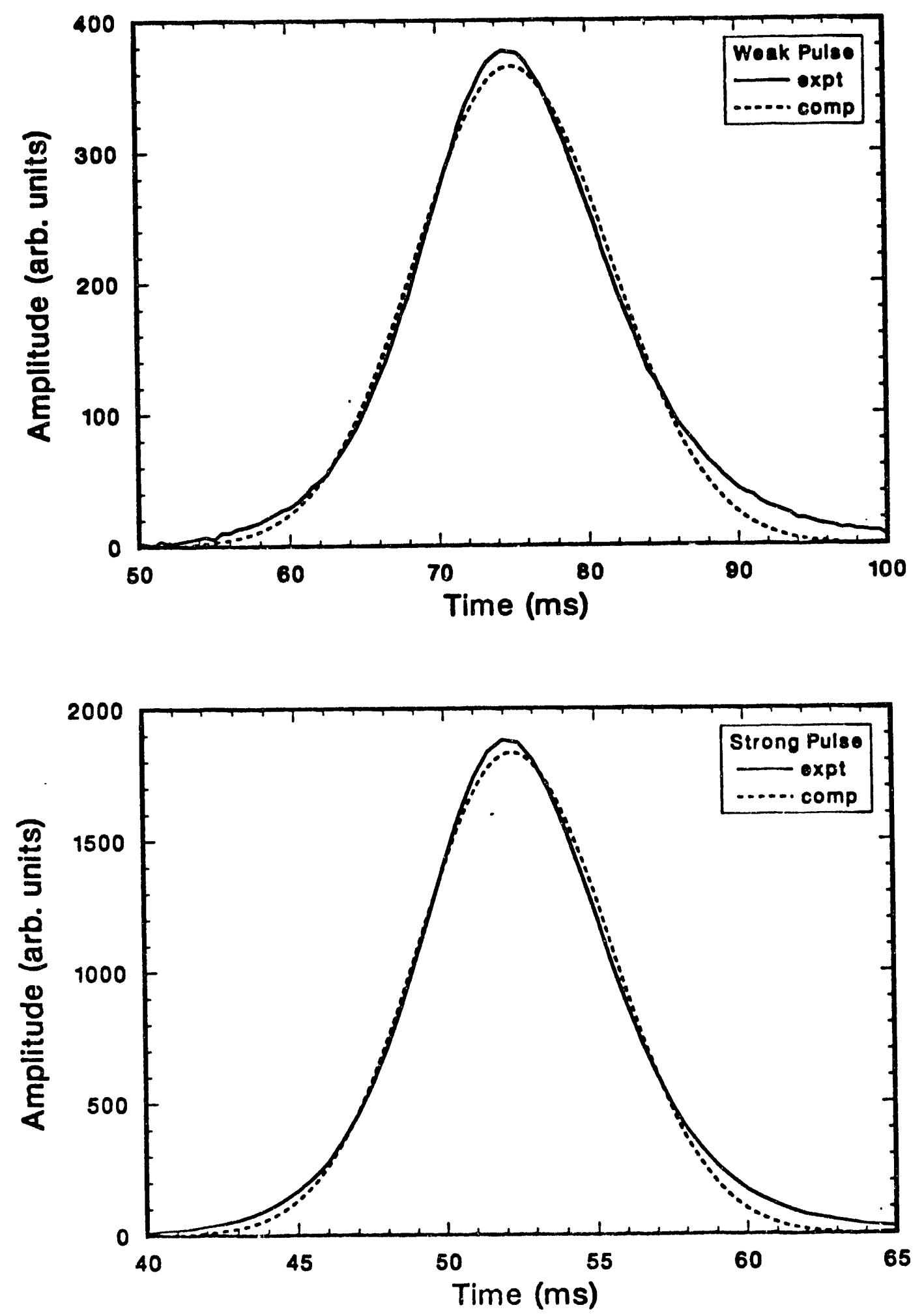

Fig. 4 Neutron flux histories (solid, expt.; dashed, model) for the weak and strong pulses. 

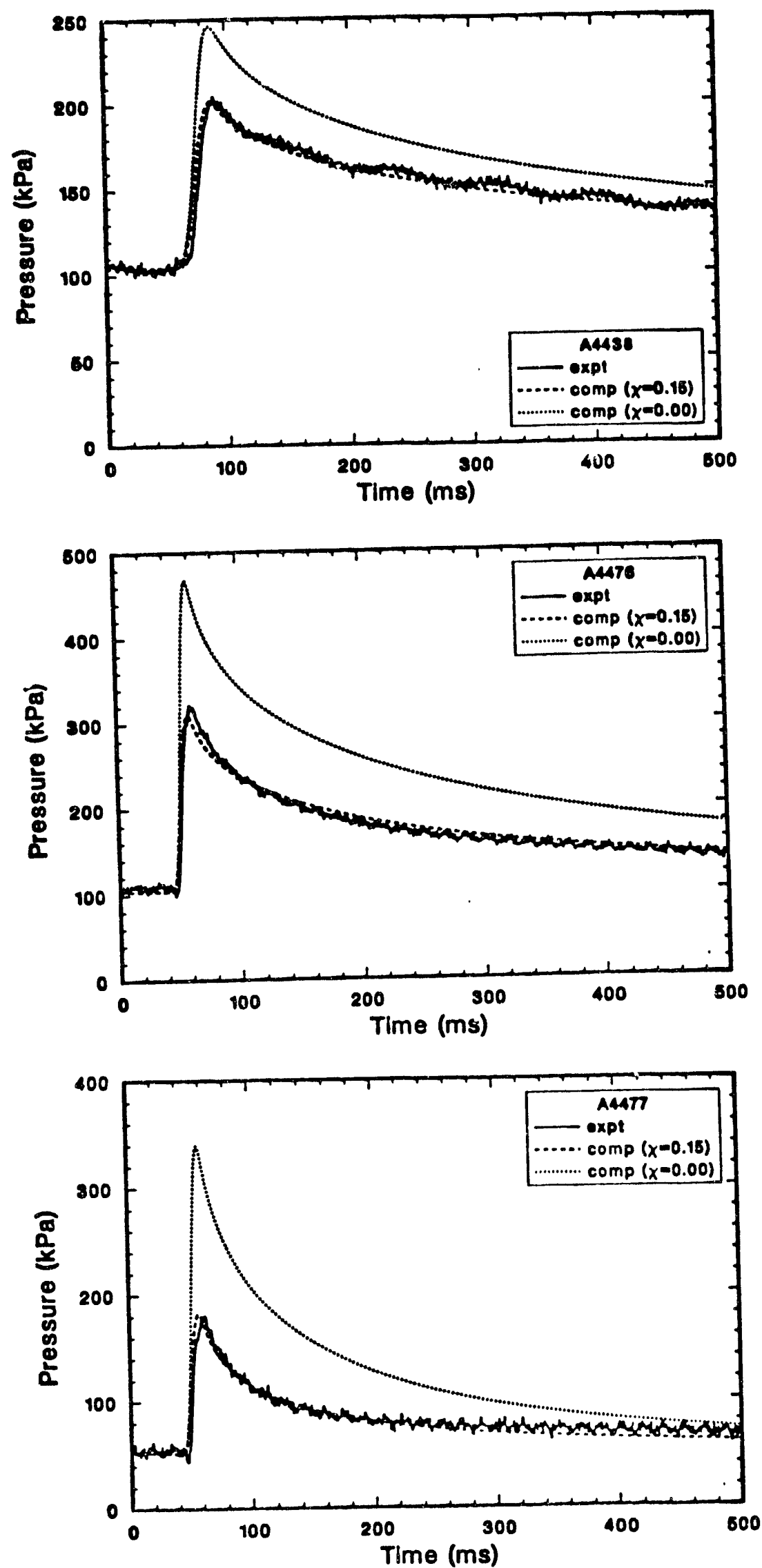

Fig. 5 Pressure histories for experiments A4438, A4476, and A4477. 

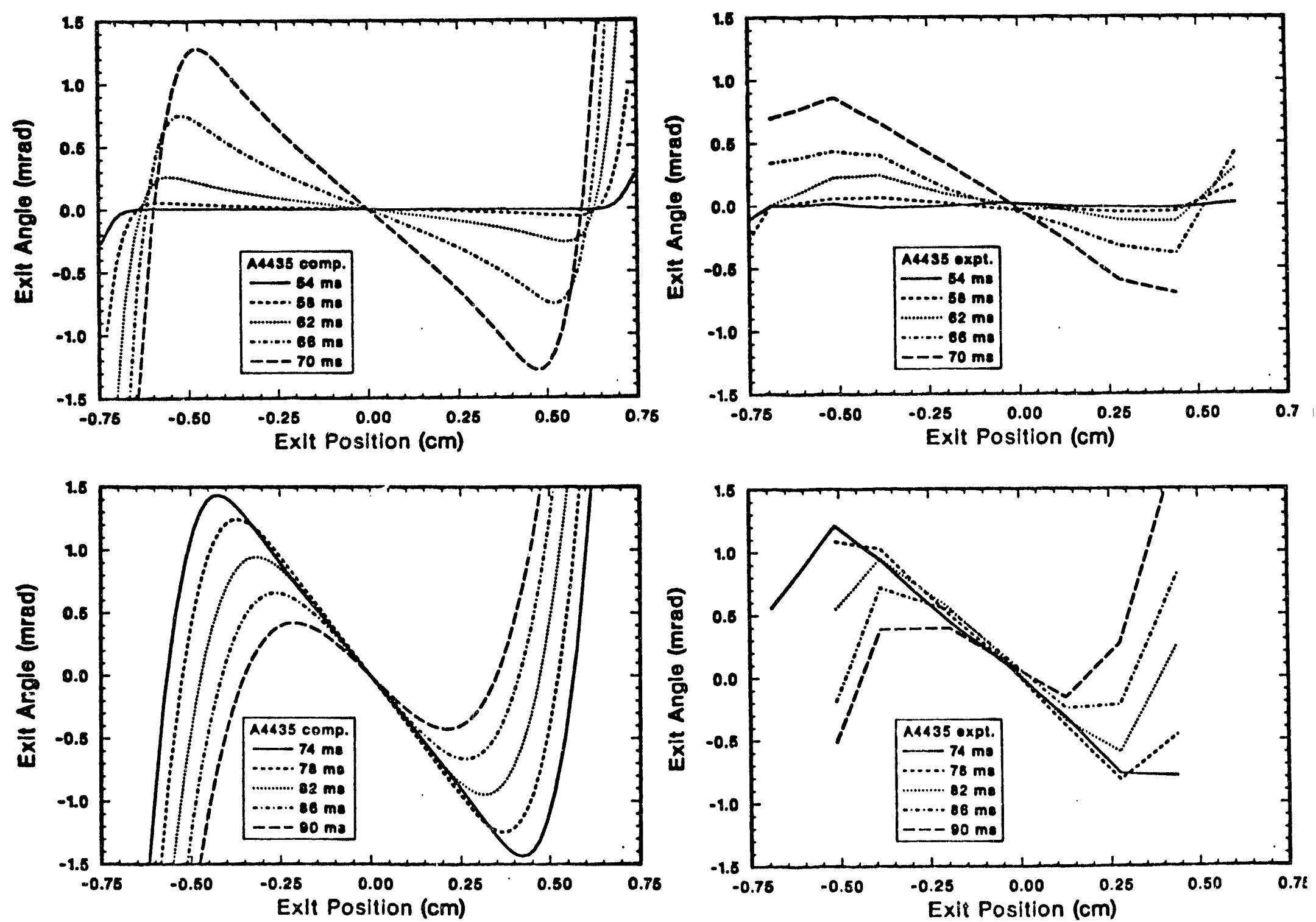

Fig. 6 Exit angle for experiment A4435. 

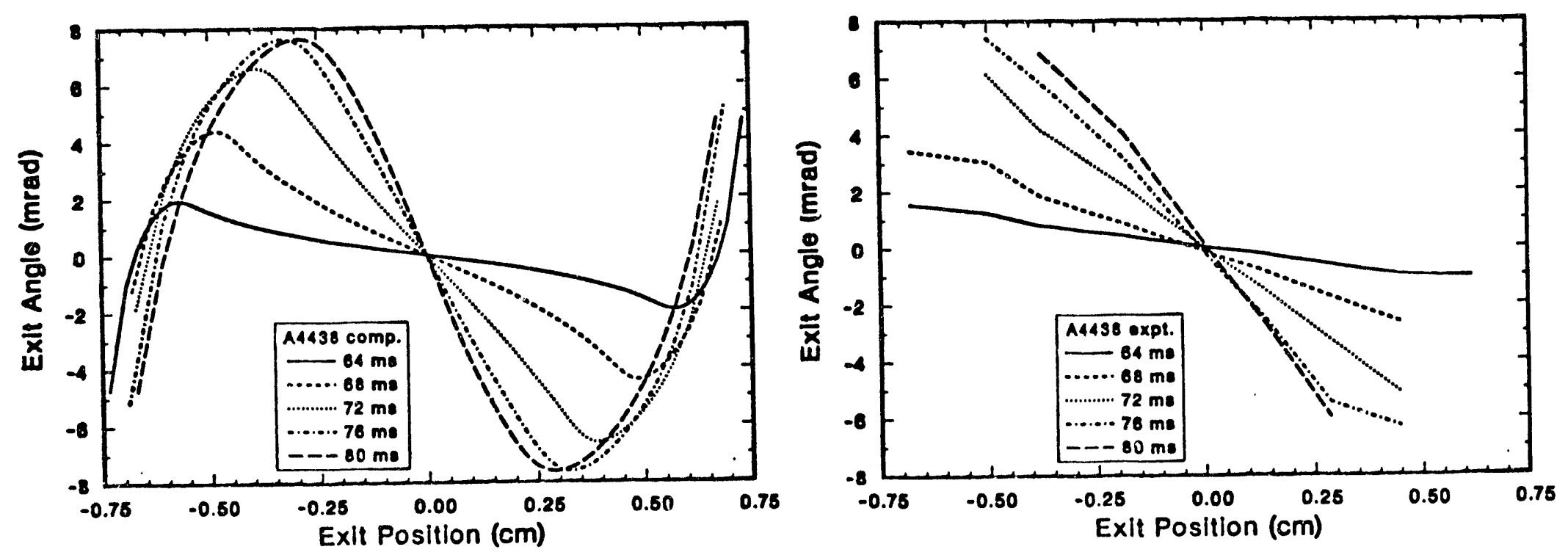

Fig. 7 Exit angle for experiment A4438. 

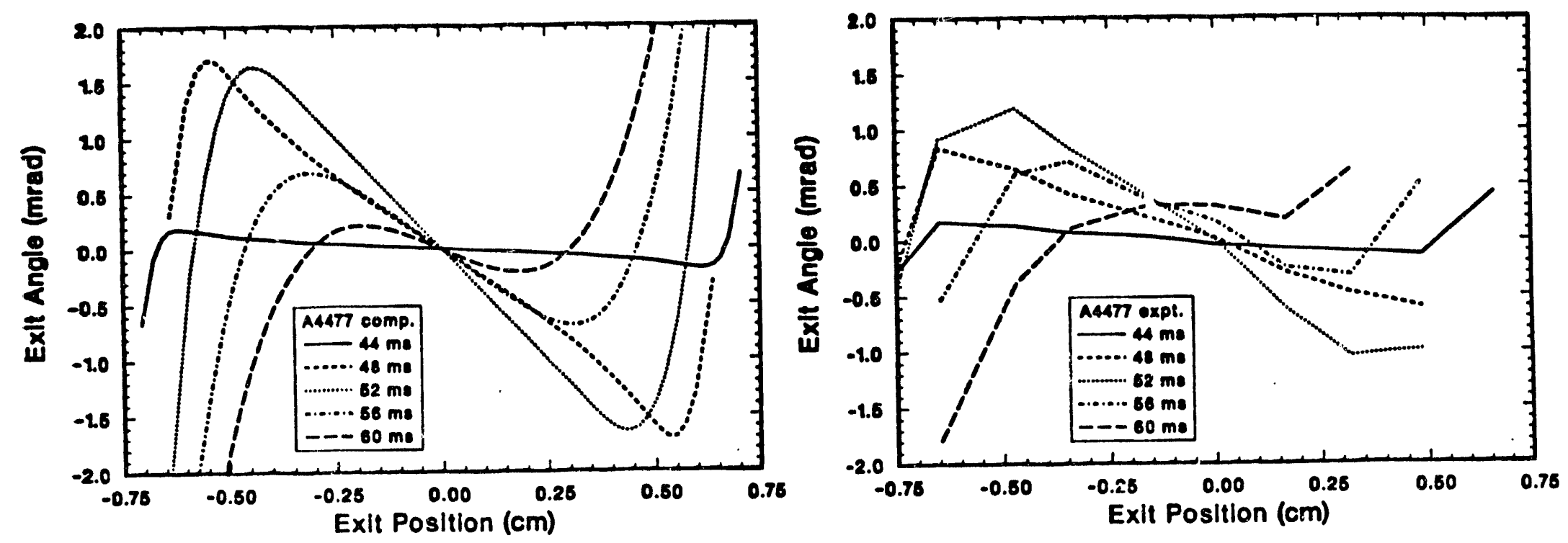

Fig. 8 Exit angle for experiment A4477.
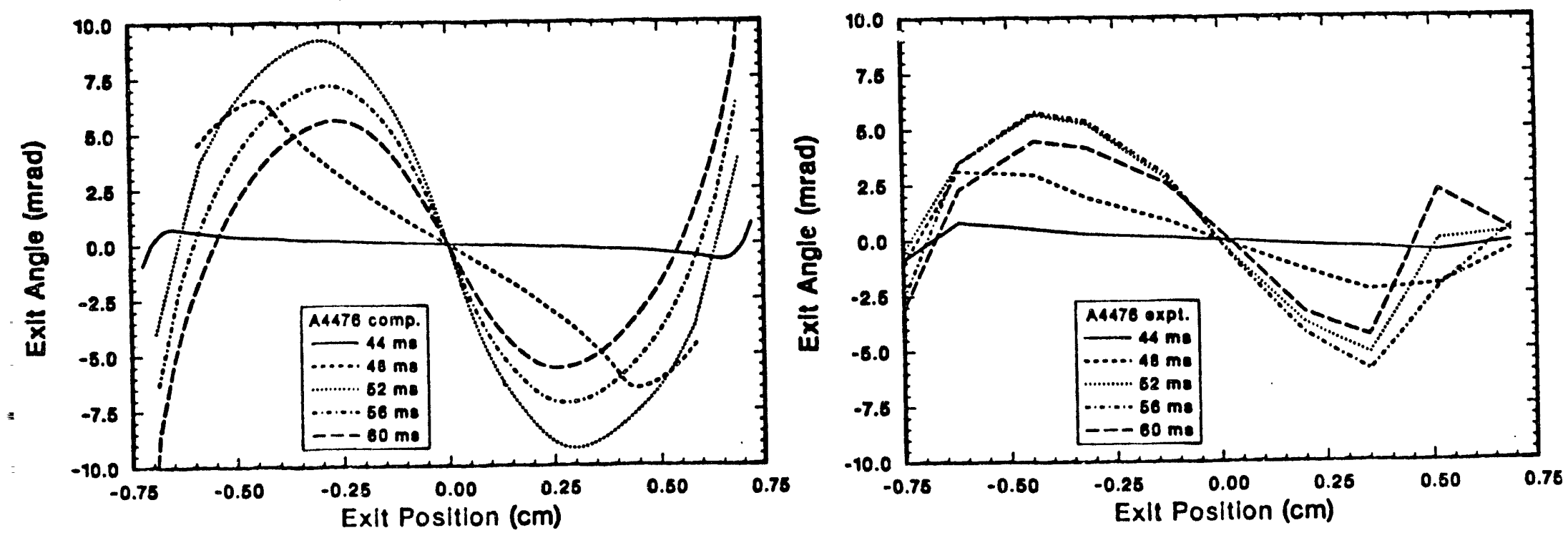

Fig. 9 Exit angle for experiment A4476. 

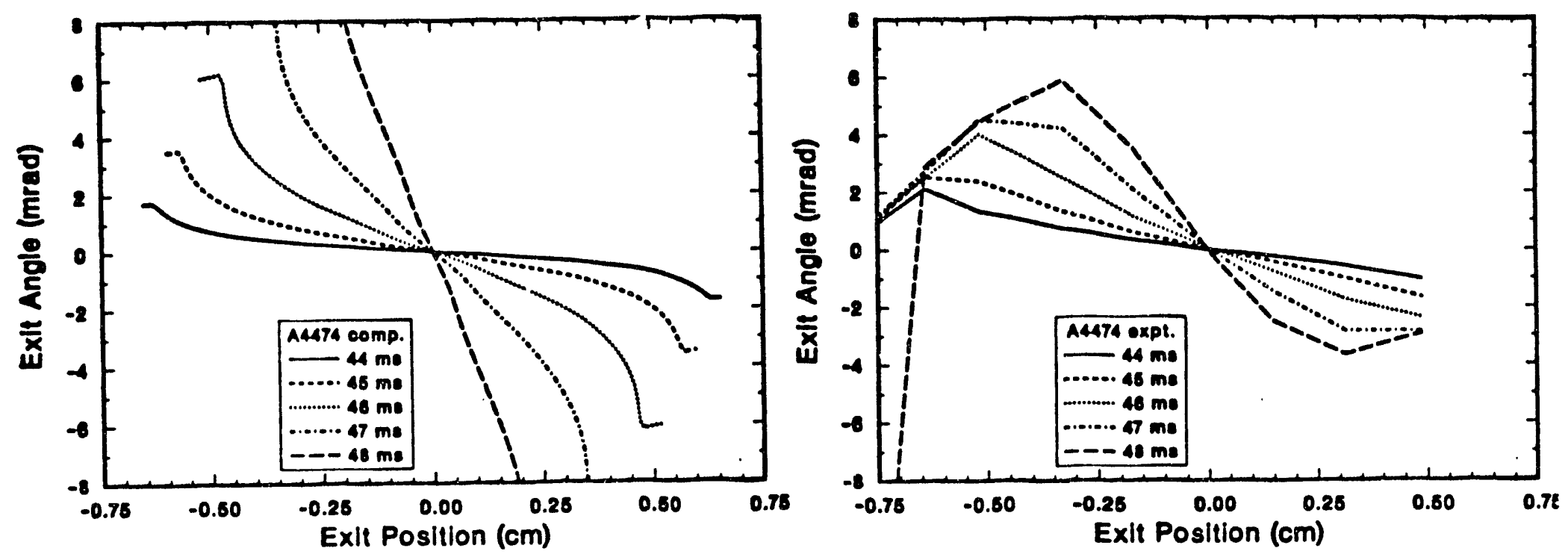

Fig. 10 Exit angle for experiment A4474.
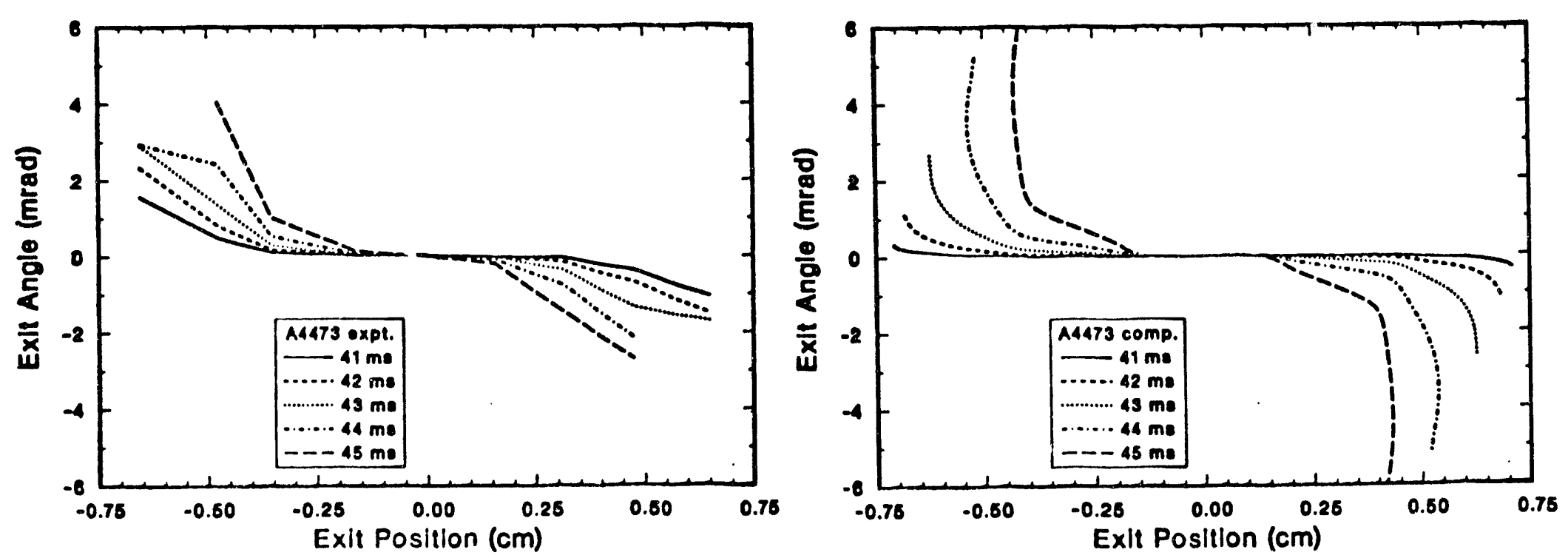

Fig. 11 Exit angle for experiment A4473. 

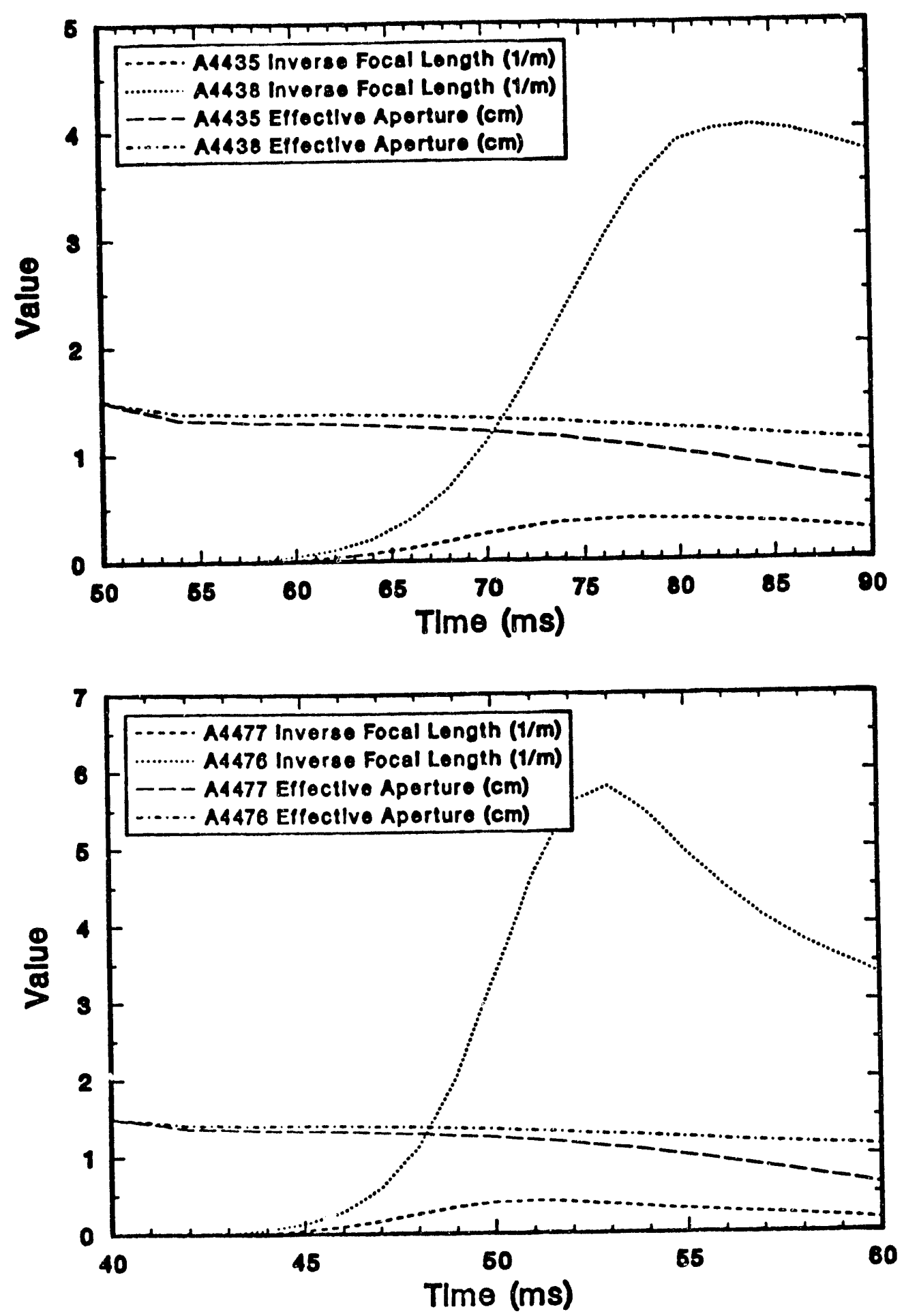

Fig. 12 Temporal variation of inverse effective focal length and effective aperture: (a) weak pulse; (b) strong pulse. 


\section{Program Announcement}

"Transient Gas Motion in Nuclear-Reactor-Pumped Lasers: Computational and Experimental Results," J. R. Torczynski and D. R. Neal, Sandia National Laboratories

Name, address, and telephone for all correspondence:

John R. Torczynski

Fluid, Thermal, and Structural Sciences Department

Division 1512

Sandia National Laboratories

Albuquerque, NM 87185-5800

(505) 845-8991 (office)

(505) 846-0295 (FAX) 

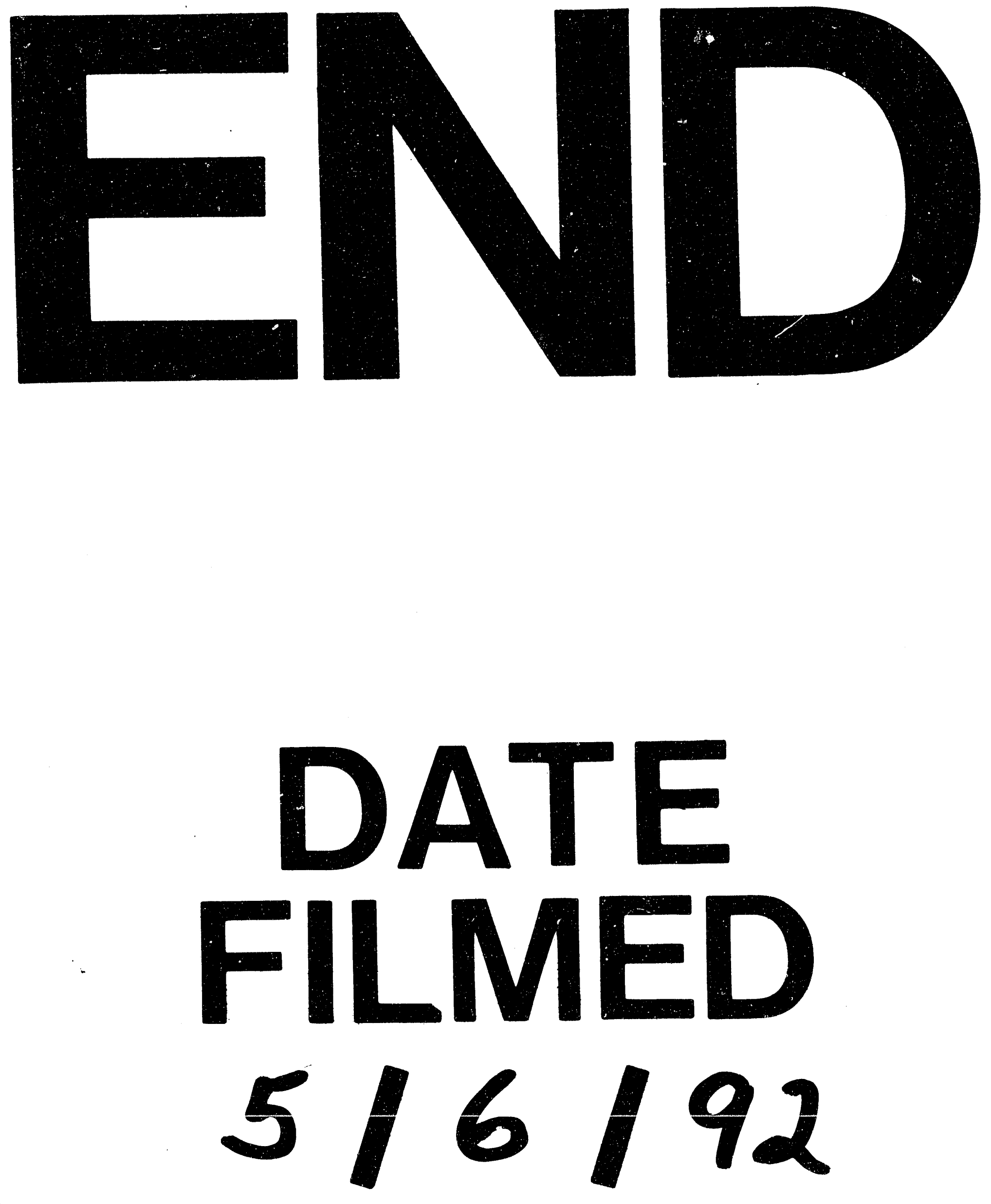

1 
